\title{
Toxicity of Hypofractionated Whole Breast Radiotherapy Without Boost in a Large "Real-Life" Cohort of Consecutive Early-Stage Breast Cancer Patients
}

\author{
Andrei Fodor ( $\nabla$ fodor.andrei@hsr.it ) \\ IRCCS Ospedale San Raffaele https://orcid.org/0000-0002-9285-4390 \\ Chiara Brombin \\ Vita-Salute San Raffaele University: Universita Vita Salute San Raffaele \\ Paola Mangili \\ IRCCS Ospedale San Raffaele \\ Roberta Tummineri \\ IRCCS Ospedale San Raffaele \\ Marcella Pasetti \\ IRCCS Ospedale San Raffaele \\ Flavia Zerbetto \\ IRCCS Ospedale San Raffaele \\ Barbara Longobardi \\ IRCCS Ospedale San Raffaele \\ Ariadna Sanchez Galvan \\ IRCCS Ospedale San Raffaele \\ Chiara L. Deantoni \\ IRCCS Ospedale San Raffaele \\ Italo Dell'Oca \\ IRCCS: IRCCS Ospedale San Raffaele \\ Paola M.V. Rancoita \\ Vita-Salute San Raffaele University: Universita Vita Salute San Raffaele \\ Claudio Fiorino \\ IRCCS Ospedale San Raffaele \\ Antonella Del Vecchio \\ IRCCS Ospedale San Raffaele \\ Mariaclelia S. Di Serio \\ Vita-Salute San Raffaele University: Universita Vita Salute San Raffaele \\ N.G. Di Muzio \\ IRCCS Ospedale San Raffaele
}

\section{Research Article}

Keywords: Radiotherapy in conservative breast cancer treatment, Toxicity of breast cancer radiotherapy, Hypofractionated radiotherapy, Breast radiotherapy boost 
Posted Date: June 2nd, 2021

DOI: https://doi.org/10.21203/rs.3.rs-559452/v1

License: (c) (i) This work is licensed under a Creative Commons Attribution 4.0 International License. Read Full License 


\section{Abstract}

Aim

To report toxicity of hypofractionated whole-breast radiotherapy (HWBRT) in a large cohort of early-stage breast cancer (BCA) patients.

Methods and Materials

From 02/2009-05/2017, 1325 consecutive BCA patients were treated with $40.05 \mathrm{~Gy} / 15$ fractions, without boost with manually optimized IMRT. Median age: 62 (IQR:51.1-70.5) years. Chemotherapy was prescribed for $28 \%$ of patients, hormonal therapy for $80.3 \%$, monoclonal antibodies for $8.2 \%$. Ten $\%$ of patients $<45$ years, $23.8 \%$ obese, $5.9 \%$ diabetic, $34 \%$ hypertensive.

Results

Median follow-up: 72.4 (IQR: 44.6-104.1) months. Acute RTOG toxicity was: 69.8\% Grade(G)1, 14.3\% G2 and $1.7 \%$ G3. Late SOMA-LENT toxicities were: edema-hyperpigmentation(E-H): G1 28.67\%, G2 4.41\%, G3 0.15\%; fibrosis-atrophytelangiectasia-pain(F-A-T-P): G1 14.6\%, G2 3.2\%, G3 0.8\%, G4 0.1\%. Median time to first occurrence was 6 and 18 months, respectively.

Aesthetic result after surgery was excellent in $28.7 \%$, good in $41.5 \%$, acceptable in $20.3 \%$ and poor in $9.5 \%$ of patients. Change in breast appearance after radiotherapy was mild in $6.9 \%$, moderate in $2.3 \%$ and marked in $1.3 \%$ of patients.

Concomitant chemotherapy, obesity, smoking, use of bolus and planning target volume (PTV) were associated with higher acute toxicity. Patients $\geq 55$ years old were less likely to experience acute toxicity. PTV and acute G2 toxicity were associated with $\geq \mathrm{G} 2 \mathrm{E}-\mathrm{H}$. PTV, concomitant chemotherapy, hypertension and $\geq \mathrm{G} 2$ acute toxicity were associated with increased risk of F-A-T-P.

Conclusion

HWBRT without boost demonstrated mild acute and late toxicity in a large cohort of consecutive patients. Timing of changes in breast appearance after radiotherapy was registered and patient, therapy and dosimetric predictive toxicity factors were identified.

\section{Introduction}

Hypofractionated whole breast radiotherapy (HWBRT) becomes the standard adjuvant treatment in BCT over the last decade, demonstrating similar risk of loco-regional failure, breast cancer death, no differences in late toxicity and decreased risk of acute toxicity, edema and telangiectasia with standard fractionation $[1,2]$. All patients of the Ontario trial and more than half of those in START-B were treated with HWBRT without boost, with good results [3, 4]. Early in 2009 our department adopted the HWBRT of the START B trial, without boost, to de-escalate the treatment and improve cosmetic outcomes, while maintaining good local control and here we report toxicity results and clinical and dosimetric factors associated with increased risk of acute and late toxicity.

\section{Material And Methods}

Study details have already been published elsewhere [5]. In short, from February 2009 to May 2017, 1325 consecutive patients who underwent BCT for early-stage breast cancer $(\mathrm{BCa})$ were treated with HWBRT. All patients were treated 
with manually optimized intensity-modulated with/without physical/dynamic wedges at $40.0 \mathrm{~Gy} / 15 \mathrm{fr}$ without boost to the tumor bed independently of age, obesity, breast volume, side, chemotherapy/trastuzumab prescription, comorbidities, and were included in this analysis. All patients signed an informed consent and the institutional ethics committee approved the study.

Significant patient and treatment characteristics for this toxicity analysis are summarized in Table 1. 
Patient characteristics

\begin{tabular}{|c|c|}
\hline Total nr. of patients & 1325 \\
\hline Age groups (years): & $133(10 \%)$ \\
\hline$<45$ & $312(23.5 \%)$ \\
\hline $45-55$ & $322(24.3 \%)$ \\
\hline $55-65$ & $558(42.1 \%)$ \\
\hline$>65$ & $62.0[51.1-70.5]$ \\
\hline \multicolumn{2}{|l|}{ Age at diagnosis (median [IQR]) } \\
\hline \multirow[t]{2}{*}{ Side: Right vs Left } & Right 640 (48.3\%) \\
\hline & Left 685 (51.7\%) \\
\hline Chemotherapy & $954(72 \%)$ \\
\hline No & $371(28 \%)$ \\
\hline Yes & $295(79.5 \%)$ \\
\hline Chemotherapy Type (371 patients) & $53(14.3 \%)$ \\
\hline Anthracycline / Anthracycline + Taxane based & $23(6.2 \%)$ \\
\hline \multicolumn{2}{|l|}{ Taxane based } \\
\hline \multicolumn{2}{|l|}{ Other (CMF, Capecitabine, etc.) } \\
\hline Trastuzumab (or trastuzumab/pertuzumab) & $1216(91.8 \%)$ \\
\hline No & $109(8.2 \%)$ \\
\hline \multicolumn{2}{|l|}{ Yes } \\
\hline Hormonal Therapy & $261(19.7 \%)$ \\
\hline No & $1064(80.3 \%)$ \\
\hline \multicolumn{2}{|l|}{ Yes } \\
\hline Breast size: & $232(17.5 \%)$ \\
\hline Small & $423(31.9 \%)$ \\
\hline Medium & $459(34.6 \%)$ \\
\hline Big & $211(15.9 \%)$ \\
\hline \multicolumn{2}{|l|}{ Very Big } \\
\hline Obesity & 968 (73.1\%) \\
\hline No & $315(23.8 \%)$ \\
\hline Yes & $42(3.1 \%)$ \\
\hline NA & \\
\hline
\end{tabular}

IQR = interquartile range, NA = not available, $\mathrm{CMF}=$ Cyclofosfamide + Methotrexate + 5-Florouracile 


\begin{tabular}{|ll|}
\hline Total nr. of patients & 1325 \\
\hline Diabetes & $1129(85.2 \%)$ \\
No & $78(5.9 \%)$ \\
NA & $118(8.9 \%)$ \\
\hline Smoke & $263(19.8 \%)$ \\
Smokers and former smokers & $1062(80.2 \%)$ \\
Non smokers & \\
\hline Alcohol consumption (declared) & $1267(95.6 \%)$ \\
No & $58(4.4 \%)$ \\
Yes & \\
\hline Hypertension & $761(57.43 \%)$ \\
No & $451(34.04 \%)$ \\
Yes & $113(8.53 \%)$ \\
NA & $1025(77.36 \%)$ \\
\hline Thyroid illnesses & $181(13.66 \%)$ \\
No & $119(8.98 \%)$ \\
Yes & \\
NA & \\
\hline IQR = interquartile range, NA = not available, CMF = Cyclofosfamide + Methotrexate + 5-Florouracile & \\
\hline
\end{tabular}

The 13 patients with synchronous bilateral BCA were treated concomitantly on both breasts.

Chemotherapy was prescribed for $28 \%$ of patients, $79.5 \%$ contained anthracyclines known to increase cardiac toxicity [6]; monoclonal antibodies, another cardiotoxic therapy [7], were prescribed for $109(8.2 \%)$ patients. Only $31(2.3 \%)$ patients with cyclofosfamide-methotrexate-5-florouracil chemotherapy (CMF) $(n=28)$ or Taxol $(n=3)$ were treated with concomitant radiotherapy, known to increase skin toxicity [8].

\section{Simulation, contouring, treatment planning, patient monitoring:}

Whole breast was the clinical target volume (CTV) defined on 5-mm axial chest CT scan images, then isotropically expanded in all directions except lung, and a $5 \mathrm{~mm}$ crop made to the body to obtain the planning target volume (PTV). Critical organs (OAR) had specific constraints [5]. Multiple segments were used within a tangential two field irradiation technique to obtain a homogeneous dose, with hotspots $\operatorname{Dmax} \leq 108 \%$. Photon( $\mathrm{X}) 6$ megavolt(MV) beams with/without wedges were used; in selected cases, X18 MV segments were added to improve dose distribution. A 1-cm water-equivalent plastic bolus was admitted in the first years to improve superficial dose distribution and was used in $313(23.6 \%)$ patients, mostly for 5-7 of 15 fractions. It was abandoned after an unpublished analysis in which an increase in toxicity was observed. Set-up errors $>5 \mathrm{~mm}$ were corrected. Patients were visited twice during the treatment, and acute toxicity was reported using the RTOG scale [9]. Follow-up visits were scheduled at 6-18-30-42-54-66 months 
after RT end. The SOMA-LENT toxicity scale was used to assess late collateral effects [10,11]. Frontal and lateral photographic images were acquired at beginning and/or end of radiotherapy and during follow-up visits. Breast size, applying a visual 4-point graded scale considering breast volume and its proportion with the patient's BMI, was scored by two observers as small, medium, large and very-large. Post-surgical deficits (asymmetry, edema, scar and/or nipple retraction) were defined at baseline. Aesthetic results (photographic) were reviewed and scored by two observers and the final score (using the Harvard 4-point scale): excellent, good, acceptable and poor [12], reached by consensus.

\section{Statistical analysis}

Median and interquartile range (IQR) were reported for continuous variables, while frequency distribution and percentages were reported for categorical variables. Ordinal logistic regression model was fitted to identify risk factors for acute toxicity (outcome variable with three toxicity thresholds: G0, G1, G2 or G3). Age, neoadjuvant and concomitant chemotherapy, hormonal therapy, monoclonal antibodies, obesity, diabetes, hypertension, smoking, alcohol consumption, presence of bolus and PTV volume were entered as independent variables in the initial model. A cubic root transformation was applied to the PTV volume to normalize its skewed distribution and, to avoid collinearity issues, V105 and V95 dosimetric parameters were not included as covariates in the model. Backward elimination method was applied to select a smaller set of relevant covariates. Binary logistic regression models were estimated to identify predictors associated with the development of breast liponecrosis and those associated with the development of $\geq$ grade $(G) 2$ late edema/hyperpigmentation $(E-H)$ toxicity within six months. Cox regression model for interval censored data was applied to identify risk factors for late fibrosis-atrophy-telangiectasia-pain (F-A-T-P) toxicity $\geq$ G2. For the latter three outcomes (breast liponecrosis, E-H and F-A-T-P) the same set of independent variables were considered as for acute toxicity outcome. We wish to highlight that for both late E-H and late F-A-T-P, the occurrence of $\geq \mathrm{G} 2$ toxicity was considered the event of interest without distinguishing among different skin reactions/manifestations.

Due to the small number of events, nonparametric tests such as Mann-Whitney test and Fisher's exact test were performed, depending on the type (quantitative/qualitative) of variable tested, to examine differences between patients with and without late cardiac or with and without lung toxicities with respect to patient and treatment characteristics, D5\%, V35 Gy, V20 Gy and V95\% or mean lung doses, D20\%, V22 Gy, V17 Gy, and mean heart doses (for left sided patients), respectively. All the analyses were performed using R statistical software (version 3.5.2, https://cran.rproject.org/index.html). Significance level was set at 0.05 .

\section{Results}

Median follow-up was 72.4 (IQR:44.6-104.1) months. A median number of 4 (2-11) segments were used. While for $90.7 \%$ of patients up to 4 fields were necessary to reach the constraints, a more complex construction was required in the remaining patients. Median PTV and OAR volumes and selected dosimetry parameters were included in Table 2. 
Table 2

Median target and OAR volumes and parameters analyzed in 1325 patients

\begin{tabular}{|ll|}
\hline Parameter & Median [IQR] \\
\hline PTV (cc) & $642.30[445.50,916.61]$ \\
\hline Target V95\% (\%) & $96.29[95.33,97.40]$ \\
\hline Target V105\% (\%) & $2.44[1.07,4.38]$ \\
\hline Body D1\% (Gy) & $41.02[40.78,41.27]$ \\
\hline Body 105\% (cc) & $28.84[13.63,53.41]$ \\
\hline Body 95\% (cc) & $1036.14[751.00,1367.76]$ \\
\hline Lung Dmean (Gy) & $5.70[4.67,6.70]$ \\
\hline Lung D20\% (Gy) & $3.72[2.84,5.67]$ \\
\hline Lung V22 Gy (\%) & $11.16[8.41,13.61]$ \\
\hline Lung V17 Gy (\%) & $12.29[9.60,15.02]$ \\
\hline Heart Dmean (Gy)* & $2.49[1.78,3.19]$ \\
\hline Heart V35Gy (cc)* & $3.76[0.59,8.94]$ \\
\hline Heart V20Gy (cc)* & $14.51[5.31,23.85]$ \\
\hline Heart V95\% (cc)* & $0.15[0.00,1.77]$ \\
\hline Heart D5\% (Gy)* & $6.74[3.68,14.77]$ \\
\hline cc = cubic centimeters, Dmean = mean dose, IQR = interquartile range. *data for left breast treatments \\
\hline
\end{tabular}

Acute toxicity according to RTOG scale was: $14.3 \% \mathrm{G} 0,69.8 \% \mathrm{G} 1,14.3 \% \mathrm{G} 2$ and $1.7 \% \mathrm{G} 3$. In $4 \%$ of patients the maximal acute toxicity grade increased in the 7 days after radiotherapy end.

Late toxicities, reported with SOMA-LENT scale, were divided into E-H (which generally improve over time) and F-A-T-P (due to their persistence over time): E-H: G0 66.77\%, G1 28.67\%, G2 4.41\%, G3 0.15\%; F-A-T-P: G0 81.3\%, G1 14.6\%, G2 $3.2 \%$, G3 $0.8 \%$, G4 $0.1 \%$. Of 141 (10.6\%) patients with liponecrosis registered, only one (0.1\%) needed surgery (G4) for the infection and subsequent fistulization.

Thirty-three (2.5\%) patients presented heart disease diagnosed during the follow-up. Seven of them received anthracycline-based chemotherapy and/or trastuzumab [6, 7]. Twenty-seven/33 patients had hormonal therapy (HT) prescription. Twelve were right-sided (1.9\% of right-sided patients) and 21 left-sided (3.1\% of left-sided patients). There was not a significant association between heart disease diagnosed and breast side $(p=0.22$, Fisher's exact test). Four patients died of heart attack, three treated on the right breast, and one on the left (with a Dmean heart $=2.1 \mathrm{~Gy}$ ). None was treated with chemotherapy or trastuzumab, all received $\mathrm{HT}$, three of them aromatase inhibitors (AI), and one switched to two years of Al after three years of Tamoxifen. Al are associated with increased cardiovascular mortality and ischemic stroke [13]. Another patient, treated with Al died of ischemic stroke.

Although pulmonary fibrosis was not systematically investigated, and was reported for only symptomatic patients or those where it was observed on CT or chest X-ray performed for other reasons, 19 patients (1.4\%) were diagnosed with a pulmonary event. 


\section{Late skin toxicity evolution}

E-H were present mainly at six-month follow-up (in approximately $33 \%$ of patients) and decreased over time.

Hence, considering only patients with complete follow-up up to 42 months $(n=998)$ the proportion of patients with $\mathrm{G0}$ toxicity increased from $68.1 \%$ at six months to $96.8 \%$ at 42 months; the proportion of patients with G1 toxicity decreased from $28-3 \%$, and G2 toxicity from 3.8-0.2\%. G3 toxicity disappeared within 18 months (see Fig. 1).

The evolution of F-A-T-P toxicity was opposite to that of E-H toxicity. Even though improvements over time were registered for some patients, an overall worsening trend for toxicity over time was observed. For those patients showing at least G1 F-A-T-P toxicity overall, the first occurrence of the toxicity was registered at 6 months for $37.4 \%$ of the patients. For the same patients the median time to first occurrence of toxicity was 18 months.

Focusing on the 998 patients with complete follow-up up to 42 months, the proportion of patients with G0 toxicity decreased from $94.4 \%$ at 6 months to $88.3 \%$ at 42 months, while the proportion of patients with $\mathrm{G} 1$ toxicity increased from $4.9-8.7 \%$, and $\mathrm{G} 2$ toxicity from $0.7-2.4 \%$. G3 toxicity was registered for the first time at 18 months after radiotherapy, increasing from 0.3 to $0.5 \%$ at 42 months, while the sole $\mathrm{G} 4$ toxicity registered at 18 months follow-up remained unchanged at 42 months (see Fig. 2). Few patients worsened thereafter, but one new G1toxicity was registered even at the 9 th-year of follow-up.

Aesthetic result after surgery, evaluated on the Harvard 4-point scale at the time of radiation treatment, was excellent in $28.7 \%$, good in $41.5 \%$, acceptable in $20.3 \%$ and poor in $9.5 \%$ of patients.

Change in breast appearance after radiotherapy was separately evaluated (patient-reported, clinically and with photographs) and at the end of follow-up was mild in $6.9 \%$, moderate in $2.3 \%$ and marked in $1.3 \%$ of patients.

\section{Other primary tumors:}

All other primary tumors observed up to the end of follow-up, regardless of time of onset during the life of the patients, were registered.

$79.2 \%$ of patients did not present any other primary tumor, $18.2 \%$ one other primary tumor, $2.4 \%$ two other primary tumors, and $0.2 \%$ three other primary tumors. Of the $20.8 \%$ of patients with another primary tumor the majority presented a previous tumor (56.7\%), $12 \%$ a concomitant tumor, $0.4 \%$ a previous and concomitant tumor, $2.9 \%$ previous and successive, $0.7 \%$ concomitant and successive, and $27.3 \%$ a successive tumor only. Of the 311 other primary tumors registered (from 1 to 3 in 275 patients) the first five in order of incidence were: $44.1 \%$ breast cancer, $14.5 \%$ digestive tumors, $11.3 \%$ gynecologic tumors, $6.8 \%$ skin tumors and $5.1 \%$ haematologic tumors. Of patients with other primary tumors $31.3 \%$ presented a family history of breast cancer. One breast angiosarcoma and one skin cancer of the irradiated breast were registered, and can be considered secondary tumors.

\section{Factors influencing acute toxicity}

In the final selected ordinal regression model, concomitant chemotherapy, obesity, smoking, presence of bolus and PTV volume were significantly associated with higher acute toxicity (G1 or G2/G3 versus G0), while patients $\geq 55$ years seem less likely to experience a higher acute toxicity (see Table 3 ). 
Table 3

Factors influencing acute toxicity

\begin{tabular}{|lll|}
\hline & OR $(95 \% \mathrm{Cl})$ & p-value \\
\hline Age $45-55$ & $0.86(0.55-1.34)$ & 0.4939 \\
\hline Age $55-65$ & $0.63(0.4-0.99)$ & 0.0466 \\
\hline Age $>=65$ & $0.54(0.35-0.82)$ & 0.0042 \\
\hline Concomitant chemotherapy & $2.24(1.03-4.9)$ & 0.0425 \\
\hline Obesity & $1.59(1.15-2.18)$ & 0.0044 \\
\hline Smoking & $1.51(1.12-2.04)$ & 0.0064 \\
\hline Bolus & $2.22(1.67-2.96)$ & $<0.001$ \\
\hline PTV (cubic root) & $1.35(1.23-1.48)$ & $<0.001$ \\
\hline
\end{tabular}

Factors influencing late toxicity: Since almost all patients experiencing $\geq \mathrm{G} 2$ late $\mathrm{E}-\mathrm{H}$ toxicity (59/60) had the event within six months, we considered the event occurrence within six months as outcome, and modelled it within a logistic regression framework. In the final selected logistic regression model, PTV volume was significantly associated with $\geq$ $\mathrm{G} 2$ late $\mathrm{E}-\mathrm{H}$ toxicity $(\mathrm{OR}=1.58,95 \% \mathrm{Cl}: 1.33-1.89$, $\mathrm{p}$-value $<0.001)$. Moreover, when considering only acute toxicity as independent predictor, for patients with acute toxicity $\geq \mathrm{G} 2$, the odds of having $\geq \mathrm{G} 2$ late E-H toxicity was 2.63 times that of patients with acute toxicity < G2 (95\% Cl: 1.49-4.64; p-value <0.001).

Considering the final selected Cox regression model for late F-A-T-P toxicity $\geq G 2$, hypertension (HR $=2.17,95 \% \mathrm{Cl}$ : 1.10-4.29, p-value $=0.0252)$, concomitant chemotherapy $(\mathrm{HR}=4.54,95 \% \mathrm{Cl}: 1.06-19.52$, $\mathrm{p}$-value $=0.0419)$ and PTV volume $(\mathrm{HR}=1.28,95 \% \mathrm{Cl}$ : $1.05-1.56, \mathrm{p}$-value $=0.0136)$ were associated with increased risk of the event, while patients $>65$ years were less likely to experience the event compared to patients $<45$ years $(\mathrm{HR}=0.27,95 \% \mathrm{Cl}: 0.1-0.76$, $\mathrm{p}$-value $=0.0132$ ). Moreover, considering only acute toxicity as independent predictor, patients with acute toxicity $\geq \mathrm{G} 2$ showed higher risk of having late F-A-T-P toxicity $\geq \mathrm{G} 2$ than patients with acute toxicity $<\mathrm{G} 2(\mathrm{HR}=2.31,95 \% \mathrm{Cl}$ : $1.33-$ 4.04; p-value $=0.0032$ ).

For liponecrosis, again referring to the final logistic regression model selected, PTV volume and smoking were significantly associated with increased toxicity risk, while HT was a protective factor (see Table 4).

Table 4

Factors influencing liponecrosis

\begin{tabular}{|lll|}
\hline & OR $(95 \% \mathrm{Cl})$ & p-value \\
\hline Hormonal therapy & $0.64(0.43-0.97)$ & 0.0339 \\
\hline Smoking & $1.62(1.09-2.42)$ & 0.0176 \\
\hline PTV (cubic root) & $1.24(1.1-1.4)$ & 0.0004 \\
\hline
\end{tabular}

Diabetes was significantly associated with late lung toxicity $(p=0.030$, Fisher's exact test), while patients with/without late cardiac toxicity differed in terms of age at diagnosis (considered as quantitative variable, $p<0.001$, Mann-Whitney test) (median age was higher in patients with cardiac toxicity).

\section{Discussion}


Despite the benefits of the shorter treatment schedule HWBRT has not been widely adopted [14]. Concerns were related to the risk of relapse but also to long term toxicity, especially in patients treated with chemotherapy, trastuzumab, left sided breast, large breast. Compared to the Ontario trial, whose patients were treated without boost, but which employed a slightly higher biologically effective dose than ours, more than $40 \%$ of START and $23 \%$ of DCGB HYPO trial patients received boost [3,4,15-17]; thus our study represents the largest "real-life" cohort of non-selected patients treated with $40 \mathrm{~Gy} / 15 \mathrm{fr}$, without boost.

Acute toxicity is less described in the randomized prospective trials. Jagsi et al., in a real-world observational study described the acute toxicity in 578 patients treated with hypofractionation: $\mathrm{G} \geq 2$ dermatitis was registered in $27.4 \%$, while moist desquamation (G3 toxicity) in 6.6\% [18]. De Santis et al., analyzed the toxicity outcome of 537 BCa patients treated with hypofractionation ( $42.4 \mathrm{~Gy} / 16 \mathrm{fr}$ ), of whom $27 \%$ received sequential boost, reporting $61.3 \%$ G1 toxicity and $20.5 \%$ G2/G3 fibrosis [19]. Only the boost was found to increase the risk of acute toxicity at multivariate analysis. With G2 RTOG toxicity of $14.3 \%$ and G3 of $1.7 \%$ our results are favorable compared to the cited studies; concomitant chemotherapy, obesity, smoke, presence of bolus and PTV were significantly associated with higher acute toxicity, while older age was protective.

Three- and five-year cosmetic outcomes in the hypofractionated arm of the Ontario trial were excellent or good in $76.8 \%$, and $76.8 \%$ of patients respectively, thus without significant deterioration after the third year [3]. Five-year G1 skin toxicity was $10 \%$ and subcutaneous tissue $29 \%$, while $\mathrm{G} \geq 2$ toxicities were $3 \%$ and $5 \%$, respectively; 10 -year skin toxicity was $24.3 \% \mathrm{G} 1,6.4 \% \mathrm{G} 2$ and $2.5 \% \mathrm{G} 3$, while subcutaneous toxicity was $40 \% \mathrm{G} 1,9.4 \% \mathrm{G} 2$ and $2.5 \% \mathrm{G} 3$ [3, 15]. Start B trial reported a marked skin reaction during the treatment in only $0.3 \%$ of patients in the hypofractionated arm and a 5 -year mild change in breast appearance in $30.8 \%$ of patients and marked in $3.0 \%$ of patients [4]. Later Haviland et al. [16] reported the following late toxicities in the hypofractionated arm: breast shrinkage at 5 years $11.4 \%$, at 10 years $26.2 \%$, breast induration $9.6 \%$ at 5 years, $14.3 \%$ at 10 years, telangiectasia $1.8 \%$ at 5 years, $4.2 \%$ at 10 years, and breast edema $4.7 \%$ at 5 years and $5.1 \%$ at 10 years. Offersen et al. reported in 926 patients treated with $40 \mathrm{~Gy} / 15 \mathrm{fr}$ ( $23 \%$ with boost) the following toxicity rates during follow-up: induration $11 \%$, telangiectasia $7 \%$, dyspigmentation $13 \%$, edema $1 \%$, scar appearance $21 \%$, and pain $4 \%$, with favorable cosmetic outcome in $81 \%$ of patients [17]. Multivariate analysis identified breast size (PTV volume) as independent risk factor. Our G2 E-H toxicity improved from $3.8 \%$ at 6 months to $0.2 \%$ at 42 months in the 998 patients with complete follow-up up to 42 months, while G2 F-A-T-P toxicity increased from $0.7-2.4 \%$. PTV volume and acute $\geq \mathrm{G} 2$ toxicity were significantly associated with the development of $\geq \mathrm{G} 2$ late $\mathrm{E}-\mathrm{H}$ within six months, while PTV volume, acute toxicity, concomitant chemotherapy, and hypertension were again identified as significant risk factors for $\geq$ G2 F-A-T-P. Older patients showed lower risk of F-A-T-P $\geq$ G-2 compared to younger patients.

Meta-analysis demonstrated that hypofractionation is associated with significantly less acute toxicity, lower breast edema and telangiectasia, and similar late cosmesis [20,21].

Radiation associated cardiac disease (five patients died of cardiac disease, three left-sided) was rare in the DBCG HYPO trial, with a similar rate (7.6\%) of patients with trastuzumab prescription [17]. In the 10-year result publication of the Start B trial, ischemic heart disease was reported in $1.5 \%$ of patients, but confirmed in only $0.7 \%$ of patients after imaging and other investigation ( $0.4 \%$ left sided), symptomatic lung fibrosis in $1.7 \%$ of patients, confirmed in $0.7 \%$, and symptomatic rib fracture in $2.2 \%$ of patients, confirmed in $0.3 \%$ (others were traumatic or due to metastases) [16]. With 6-year median follow-up the incidence of contralateral breast cancer was $1.5 \%$ for hypofractionated arm and $2.3 \%$ of other primary cancers (lung, endometrial, ovarian, colon) [4]. Only two cases of radiation pneumonitis and one rib fracture were reported by Whelan et al $[3,15]$. Incidence of heart disease and lung pneumonitis was higher in our cohort, likely due to the higher proportion of patients with chemotherapy and Al prescription, as well as to the trastuzumab therapy in Her2 + patients, not used in the older series of Ontario and START trials. Radiotherapy may be expected to 
increase cardiac and lung toxicities, as well as second tumor incidence, and the association of synergic systemic therapies from this point of view increases the risk [22-24]. Recent reports, however, demonstrate a positive impact of new schedules and technological improvements, lowering risk $[25,26]$. The short follow-up for cardiac side effects, but also the low heart Dmean (2.49 Gy) obtained in our left-sided patients probably contributed to the low cardiac toxicity observed, with no significant difference between patients for right and left breast cancer. No rib fractures were registered in our cohort. Other primary tumors were largely discussed in results.

Major limitations of our study are the absence of randomization and its retrospective nature. The greatest strength is the homogeneity of our hypofractionated treatment, from the point of view of fractionation, dose, volumes and technical modalities, resulting in the largest cohort of "real-life" consecutive BCa patients without boost irradiation, systematically monitored for a "sufficiently long" period of time after the treatment. We observe that, despite the use of chemotherapy and trastuzumab in all "fit" patients, molecular subtypes were the most significant factors predicting relapse [5]. In these consecutive, unselected patients, treated with anthracyclines, taxanes, trastuzumab, as required by their illness, we could also evaluate acute and late toxicities and the factors influencing them. Thus we consider that, even with its limitations, this study offers "real-life" information of outcomes of patients treated with a three-week hypofractionated schedule that could be complementary to the randomized trials in which some of these treatments were less prescribed or unavailable, and could favor the wider adoption of a still underused hypofractionation [14].

Another relative limitation is the forward planned-IMRT technique. However, this approach is still widely used for breast cancer, and provides, as reported here, very good results in terms of dose homogeneity and OAR sparing. Of note, this approach was recently updated with the implementation of an automatic planning technique "in-house" developed, mimicking and slightly improving the performance, and reducing dependence on the human factor (critical in forward IMRT optimization) [27].

Results of FAST and FAST-Forward trials were recently published [28,29] and, combined with recent international recommendations to reduce hospital visits during Sars-Cov2 pandemic [30], could move treatments towards only 5 fractions. Thus the three-week schedule could become obsolete, at least for a fraction of patients. However, for the FAST-Forward trial, long-term control and toxicity data, vital for final clinical acceptance (due to the 18.8\% EQD2 dose reduction and the substantially higher dose per fraction [17]), are missing. Moreover, the boost proposed in FAST Forward trial is only sequential, reducing the overall treatment time gain, while for moderate hypofractionation a simultaneous boost can be easily integrated.

\section{Conclusion}

The HWBRT, without boost, after BCS demonstrated limited acute and late toxicity in a large real-life cohort of 1325 consecutive patients. Predictive factors for toxicity were identified in these patients treated with modern chemotherapy, trastuzumab, and a wide use of Al for a longer period than HT in the Ontario and START trials. Changes in breast appearance after radiotherapy, assessed on photographs separately from changes produced by surgery, involved $10.5 \%$ of patients, but were marked in only $1.3 \%$.

\section{Declarations}

Conflict of interests: The authors report no conflict of interests.

Funding: This research did not receive any specific grant from funding agencies in the public, commercial, or not-forprofit sectors. 
Clinical trial information:

The study is registered with ClinicalTrials.gov (NCT03077191) together with an ongoing prospective study of quality of life.

Data Sharing Statement:

The study protocol did not include a sharing plan. However, anonymized individual participant data will be available following the publication of the article until the closure of the study upon request on a case-by-case basis to researchers who provide a methodologically sound proposal. Requests made to the corresponding author will be evaluated by the IRCCS San Raffaele Scientific Institute Ethics Committee.

Acknowledgments:

The authors thank Aniko Maria Deli, MD, Department of Radiation Oncology, IRCCS San Raffaele Scientific Institute, Milan, Italy, Roberta Castriconi and Pier Giorgio Esposito, MSc's, Medical Physics, IRCCS San Raffaele Scientific Institute, Milan, Italy, for their assistance.

\section{References}

1. Agarwal S, Herchek H, Nalepinski D, Bickel-MacPhee K, Kapadia NS. Meta-analysis of standard versus hypofractionated whole-breast radiation therapy as part of breast conserving therapy for early-stage breast cancer. Int J Radiat Oncol Biol Phys; 2015: 93 (3), Suppl.: E15.

2. Andrade TRM, Fonseca MCM, Segreto HRC, Segreto RA, Martella E, Nazario ACP. Meta-analysis of long-term efficacy and safety of hypofractionated radiotherapy in the treatment of early breast cancer. Breast; 2019: 48: 2431.

3. Whelan T, MacKenzie R, Julian J et al. Randomized trial of breast irradiation schedules after lumpectomy for women with lymph node negative breast cancer. J Natl Cancer Inst 2002; 94:1143-1150.

4. The START Trialists' Group: The UK standardization of Breast radiotherapy (START) Trial B of radiotherapy hypofractionation for treatment of early breast cancer: a randomized trial. Lancet 2008;371:1098-107.

5. Fodor A, Brombin C, Mangili P et al. Impact of molecular subtype on 1325 early-stage breast cancer patients homogeneously treated with hypofractionated radiotherapy without boost: Should the indications for radiotherapy be more personalized? Breast 2021; 55:45-54.

6. Smith LA, Cornelius VR, Plummer CJ, Levitt G, Verrill M, Canney P, et al. Cardiotoxicity of anthracycline agents for the treatment of cancer: systematic review and meta-analysis of randomised controlled trials. BMC Cancer. 2010;10:337.12.

7. Yu AF, Yadav NU, Lung BY, Eaton AA, Thaler HT, Hudis CA, et al. Trastuzumab interruption and treatment-induced cardiotoxicity in early HER2-positive breast cancer. Breast Cancer Res Treat. 2015;149(2):489-95.

8. Toledano A, Garaud P, Serin D et al. Concurrent administration of adjuvant chemotherapy and radiotherapy after breast-conserving surgery enhances late toxicities : Long-term results of the ARCOSEIN multicenter randomized study. Int. J Radiat Oncol Biol Phys 2006 ; 65 (2) : 324-332.

9. https://www.rtog.org/ResearchAssociates/AdverseEventReporting/CooperativeGroupCommonToxicityCriteria.aspx

10. Mornex F, Pavy JJ, Denekamp J, Bolla M. Scoring system of late effects of radiations on normal tissues: the SOMA-LENT scale. Cancer Radiother 1997, 1(6). 622-668. 
11. Hoeller U, Tribius S, Kuhlmey A, et al. Increasing the rate of late toxicity by changing the score? A comparison of RTOG/EORTC and LENT/SOMA scores. Int J Radiat Oncol Biol Phys 2003; vol 55(4):1013-1018.

12. Harris JR, Levene MB, Svensson G, Hellman S. Analysis of cosmetic results following primary radiation therapy for stages I and II carcinoma of the breast. Int J Radiat Oncol Biol Phys.1979;5:257-61.

13. Khosrow-Khavar F, Filion KB, Bougamin N, Suissa S, Azoulay L. Aromatase inhibitors and the risk of cardiovascular outcomes in women with breast cancer. A population-based cohort study. Circulation, 2020; 141(7): 549-559.

14. Prades J, Algara M, Espinas JA, et al. Understanding variations in the use of hypofractionated radiotherapy and it specific indications for breast cancer: a mixed-methods study. Radiother Oncol 2017; 123:22-28.

15. Whelan TJ, Pignol JP, Levine MN, et al. Long-term results of hypofractionated radiation therapy for breast cancer. $\mathrm{N}$ Engl J Med 2010;362:513-20.

16. Haviland JS, Owen RJ, Dewar JA, et al. The UK Standardisation of Breast Radiotherapy (START) trials of radiotherapy hypofractionation for treatment of early breast cancer: 10-year follow-up results of two randomised controlled trials. Lancet Oncol 2013; 14: 1086-1094.

17. Offersen BV, Alsner J, Nielsen HM et al. Hypofractionated versus standard fractionated radiotherapy in patients with early breast cancer or ductal carcinoma in situ in a randomized phase III trial: the DBCG HYPO trial. J Clin Oncol 2020; 38(31): 3615-3625.

18. Jagsi R, Griffith KA, Boike TP et al. Differences in the acute toxic effects of breast radiotherapy by fractionation schedule. Comparative analysis of physician-assessed and patient-reported outcomes in a large multicenter cohort. JAMA Oncol 2015; 1(7):918-930.

19. De Santis MC, Bonfantini F, Di Salvo F, et al. Factors influencing acute and late toxicity in the era of adjuvant hypofractionated breast radiotherapy. The Breast 2016 (29):90-95.

20. Andrade TRM, Fonseca MCM, Segreto HRC, Segreto RA, Martella E, Nazario ACP. Meta-analysis of long-term efficacy and safety of hypofractionated radiotherapy in the treatment of early breast cancer. The Breast 2019; 48:24-31.

21. Valle LF, Agarwal S, Bickel KE, Herchek H, Nalepinski DC, Kapadia NS. Hypofractionated whole breast radiotherapy in breast conservation for early-stage breast cancer: a systematic review and meta-analysis of randomized trials. Breast Cancer Res Treat 2017; 162: 409-417.

22. Darby SC, McGale P, Taylor CW, Peto R. Long-term mortality from heart disease and lung cancer after radiotherapy for early breast cancer: prospective cohort study of about 300000 women in US SEER cancer registries. Lancet Oncol 2005;6: 557-65.

23. Ramin C, Schaeffer ML, Zeng Z et al. All-causes cardiovascular disease mortality among breast cancer survivors in CLUE II, a long standing community-based cohort. J Natl Cancer Inst 2021; 113(2): 137-145.

24. Grantzau T, Overgaard J. Risk of second non-breast cancer after radiotherapy for breast cancer: A systematic review and meta-analysis of 762,468 patients. Radiother Oncol 2015 (114):56-65.

25. Lee BM, Chang JS, Kim SY, Keum KC, Suh CO, Kim YB. Hypofractionated radiotherapy dose scheme and application of new techniques are associated to a lower incidence of radiation pneumonitis in breast cancer patients. Front Oncol 2020; 10: 124.

26. Milo MLH, Thorsen LBJ, Johnsen SP, et al. Risk of coronary artery disease after adjuvant radiotherapy in 29,662 early breast cancer patients : A population-based Danish Breast Cancer Group study. Radiother Oncol 2021 ; 157 : 106-113.

27. Esposito PG, Castriconi R, Mangili P, et al. Virtual Tangential-fields Arc Therapy (ViTAT) for whole breast irradiation: Technique optimization and validation. Phys Med 2020; 77:160-168. 
28. Brunt MA, Haviland JS, Sydenham M, et al. Ten-year results of FAST: A randomized controlled trial of 5-fraction whole-breast radiotherapy for early breast cancer. J Clin Oncol 2020; 38:3261-3272.

29. Brunt AM, Haviland JS, Wheatley D, et al. Hypofractionated breast radiotherapy for 1 week versus 3 weeks (FASTForward): 5-year efficacy and late normal tissue effects results from a multicentre, non-inferiority, randomised, phase 3 trial. Lancet 2020; 395: 1613-26.

30. Coles CE, Aristei C, Bliss J, et al. International Guidelines on Radiation Therapy for Breast Cancer During the COVID19 Pandemic. Clin Oncol 2020; 32: 279-281.

\section{Figures}
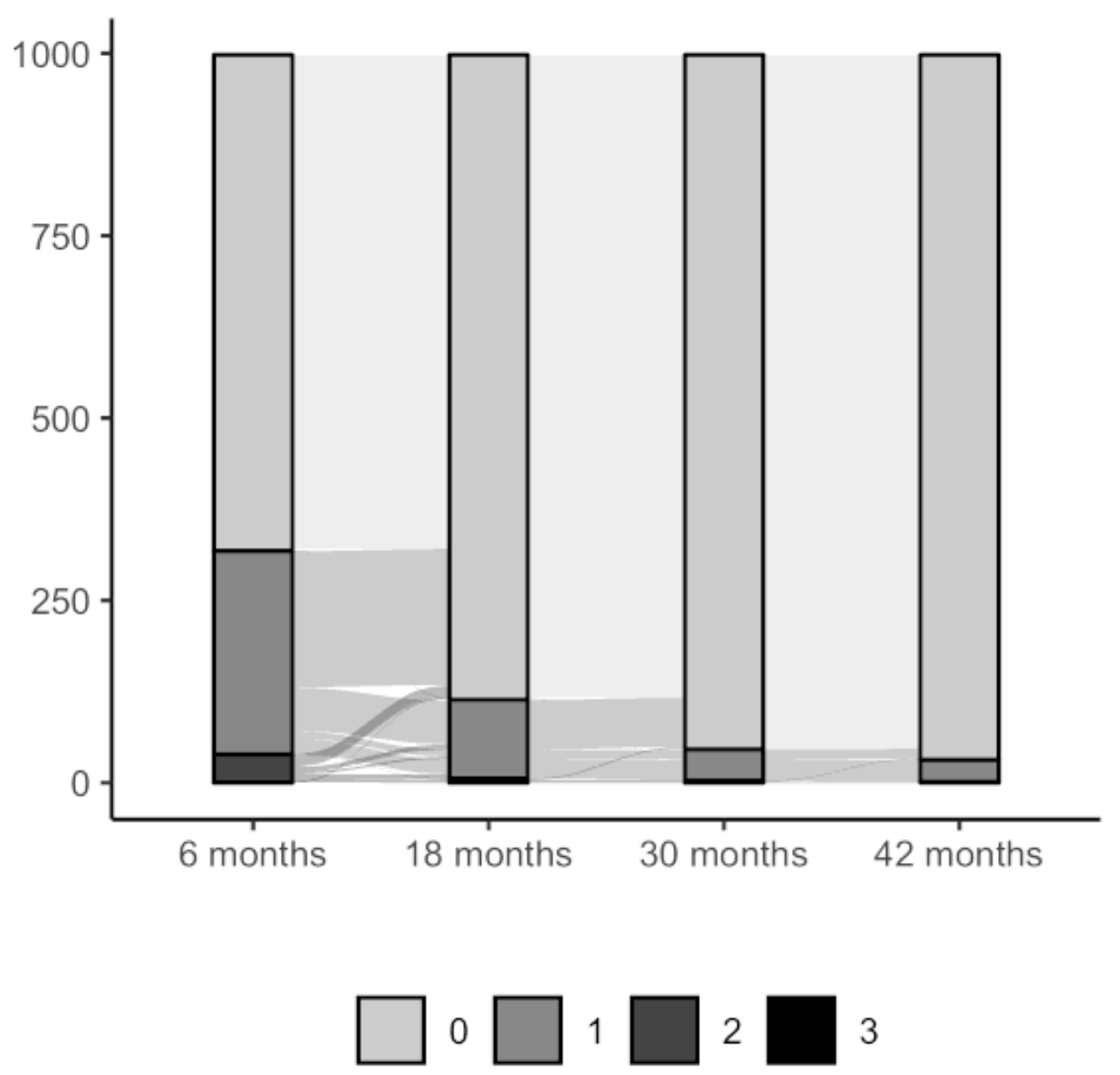

\section{Figure 1}

The decrease of late edema-hyperpigmentation over time: the alluvial plot shows the changes in degrees toxicity over time; streams are represented by the waves in shades of gray between the columns (passage from G1, G2, and G3 to $\mathrm{G0}$, from G0, G2 and $\mathrm{G} 3$ to $\mathrm{G} 1$, etc.), where the toxicity frequency distribution is shown. 


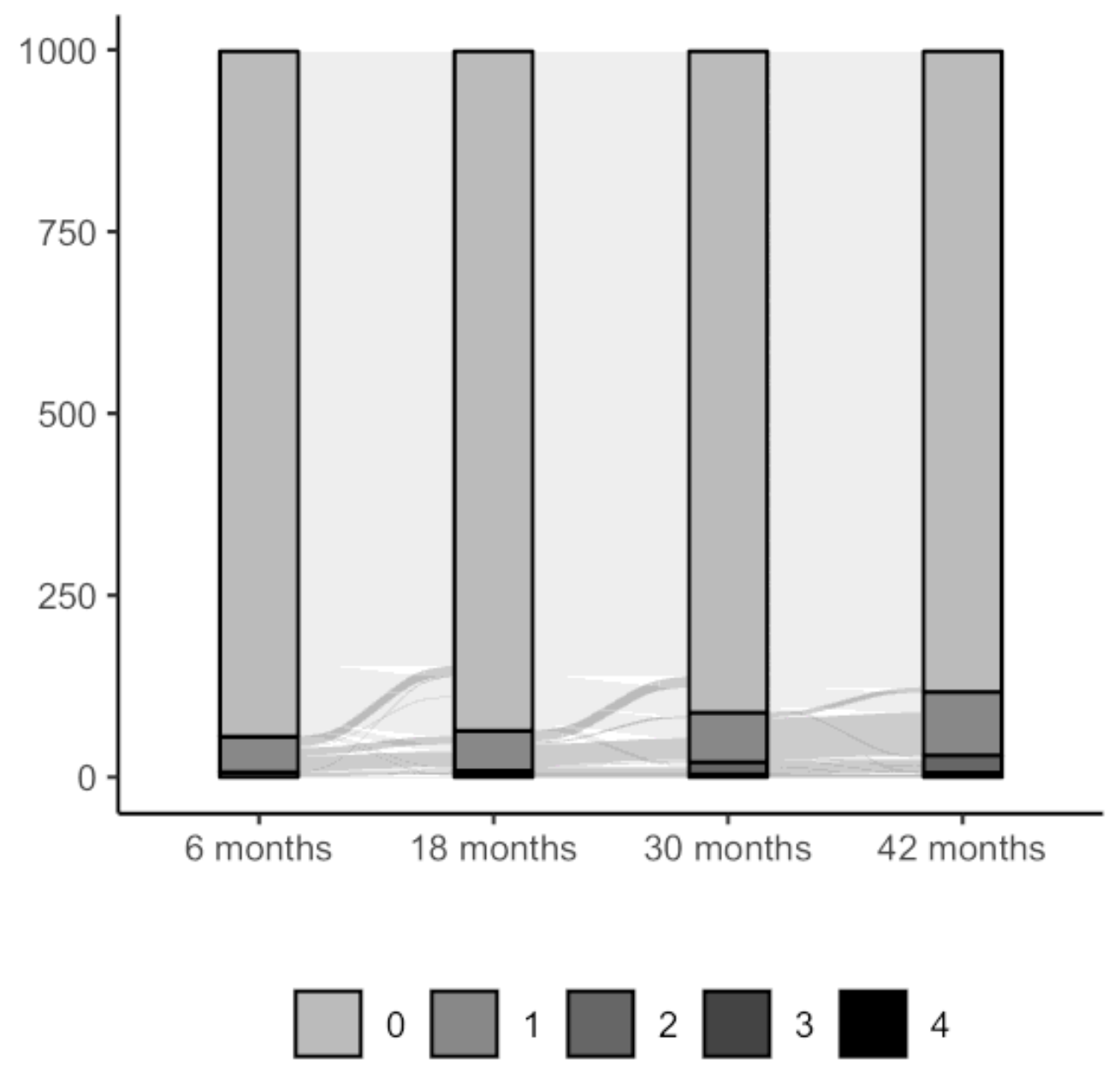

Figure 2

Increase in fibrosis-atrophy-telangiectasia-pain over time: the alluvial plot shows the changes in degrees of toxicity over time (passage from G0, to G1, G2, G3 toxicity, from G1 to G0, G2, and G3 toxicity, from G2 to G1, G3, and G4 toxicity, etc); streams are represented by waves in shades of gray between the columns, where toxicity frequency distribution is shown. 